\title{
DEVELOPMENT OF A COMPACT RADIOGRAPHY ACCELERATOR USING DIELECTRIC WALL ACCELERATOR TECHNOLOGY
}

S. Sampayan, G. Caporaso, Y-J. Chen, S. Hawkins, C. Holmes, M. Krogh, J. McCarrick, S. Nelson, W. Nunnally, B. Poole, M. Rhodes, D. Sanders, K. Selenes, J. Sullivan, L. Wang, J. Watson

June 6, 2005

IEEE International Pulsed Power Conference Monterey, CA, United States June 13, 2005 through June 17, 2005 
This document was prepared as an account of work sponsored by an agency of the United States Government. Neither the United States Government nor the University of California nor any of their employees, makes any warranty, express or implied, or assumes any legal liability or responsibility for the accuracy, completeness, or usefulness of any information, apparatus, product, or process disclosed, or represents that its use would not infringe privately owned rights. Reference herein to any specific commercial product, process, or service by trade name, trademark, manufacturer, or otherwise, does not necessarily constitute or imply its endorsement, recommendation, or favoring by the United States Government or the University of California. The views and opinions of authors expressed herein do not necessarily state or reflect those of the United States Government or the University of California, and shall not be used for advertising or product endorsement purposes. 


\title{
DEVELOPMENT OF A COMPACT RADIOGRAPHY ACCELERATOR USING DIELECTRIC WALL ACCELERATOR TECHNOLOGY*
}

\author{
S. Sampayan, G Caporaso, Y-J. Chen, S. Hawkins, C. Holmes, M. Krogh ${ }^{\mathrm{a}}$, J. McCarrick, \\ S. Nelson, W. Nunnally ${ }^{\mathrm{b}}$, B. Poole, M. Rhodes, D. Sanders, K. Selenes ${ }^{\mathrm{c}}$, J. Sullivan, \\ L. Wang, and J. Watson, \\ Lawrence Livermore National Laboratory \\ P.O. Box 808, L-645 \\ Livermore, CA 94551, USA
}

\section{Abstract}

We are developing an inexpensive compact accelerator system primarily intended for pulsed radiography. Design characteristics are an $8 \mathrm{MeV}$ endpoint energy, $2 \mathrm{kA}$ beam current, a cell gradient of approximately $3 \mathrm{MV} / \mathrm{m}$ (for an overall accelerator length is 2-3 $\mathrm{m}$ ), and $<\$ 1 /$ Volt capital costs. Such designs have been made possible with the development of high specific energy dielectrics $\left(>10 \mathrm{~J} / \mathrm{cm}^{3}\right)$, specialized transmission line designs and multi-gap laser triggered low jitter $(<1 \mathrm{~ns})$ gas switches. In this geometry, the pulse forming lines, switches, and insulator/beam pipe are fully integrated within each cell to form a compact, stand-alone, stackable unit. We detail our research and modeling to date, recent high voltage test results, and the integration concept of the cells into a radiographic system.

\section{I.INTRODUCTION}

For the past several years, Lawrence Livermore National Laboratory has been developing compact accelerator technology for radiography. This effort, funded under the US Department of Energy, has as its objective the production of a 20-30 ns, 2 kA electron beam pulse, at a $10-20 \mathrm{MV} / \mathrm{m}$ gradient. Successfully meeting such objectives would allow the implementation of an equivalent FXR or DARHT accelerator in a 1-2 meter length structure. With advances in SiC switching technology, such a system would be capable of $\mathrm{MHz}$ repetition rates and would provide a multiframe radiography capability in a compact inexpensive package.

As the first step in implementation, we have more recently focused on development of a compact system for U1-a underground radiography [1]. The objective of this system is a single $10 \mathrm{Rad}$ radiographic pulse, with a $1 \mathrm{~mm}$ spotsize and an $8 \mathrm{MeV}$ endpoint energy. Projected cost for the system were $\$ 7.6 \mathrm{M}$ or $<\$ 1 /$ volt, exclusive of the existing development program.

Classical pulsed-power approaches were ruled out because of (1) the diode technology for the required endpoint energy has not been demonstrated, (2) the background radiation generated by the vacuum power feeds and their adverse affect on the radiographic signal to noise ratio, and (3) space constraints. And although gradients for the system are not specified, the confined space of the underground facility required gradients to be maintained as high as possible.
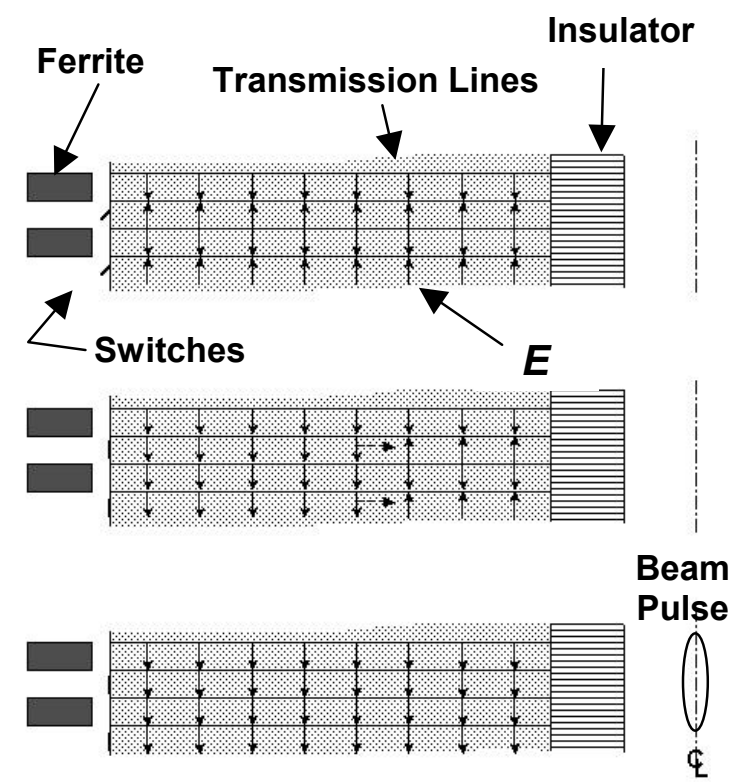

Figure 1. Symmetric Blumlein operation. Top: charging cycle. Middle: switches close to generate reverse polarity wave. Lower: beam injected and accelerated until transmission line is discharged. Ferrite isolates the leakage current resulting from the unswitched line.

\footnotetext{
* This work was supported under the auspices of the US Department of Energy, the University of California, Lawrence Livermore National Laboratory under Contract No. W-7405-Eng-48.
} 
During this phase of the project, we focused on techbase development and demonstrating the utility of the structure at high voltage. For the tech-base development, we focused on (1) insulators, (2) pulse-forming lines and dielectric materials, and (3) switching. By necessity, we also focused on code and full 3-d time dependent model development to speed up the design process with a minimum of fabrication [2]. And lastly, we performed high voltage demonstration experiments in preparation for accelerator cell construction.

There are multiple configurations for the Dielectric Wall Accelerator (DWA). The first implementation was based on the asymmetric Blumlein [3]. Although this configuration does not require magnetic material for suppression of a reflected back wave, energy transfer to the load is inefficient. For the symmetric Blumlein (fig 1), energy transfer to the beam can approach $100 \%$, but a backward wave must be suppressed to ensure full voltage erection of the structure.

\section{II.ACCELERATOR DEVELOPMENT}

The overall layout of the accelerator is shown in Figure 2 and consists of an injector composed of multiple symmetric Blumleins. Ferrite isolation is unnecessary in the injector as the geometry allows the beam current to dominate. In the present design, the injector provides an energy gain of $1.5 \mathrm{MeV}$ and the remaining 13 cells provide $6.5 \mathrm{MeV}$ for a final end-point energy of $8 \mathrm{MeV}$. Overall length from the cathode to the end of the accelerator is approximately $2-3 \mathrm{~m}$. The drift section for our particular application is approximately $3 \mathrm{~m}$.

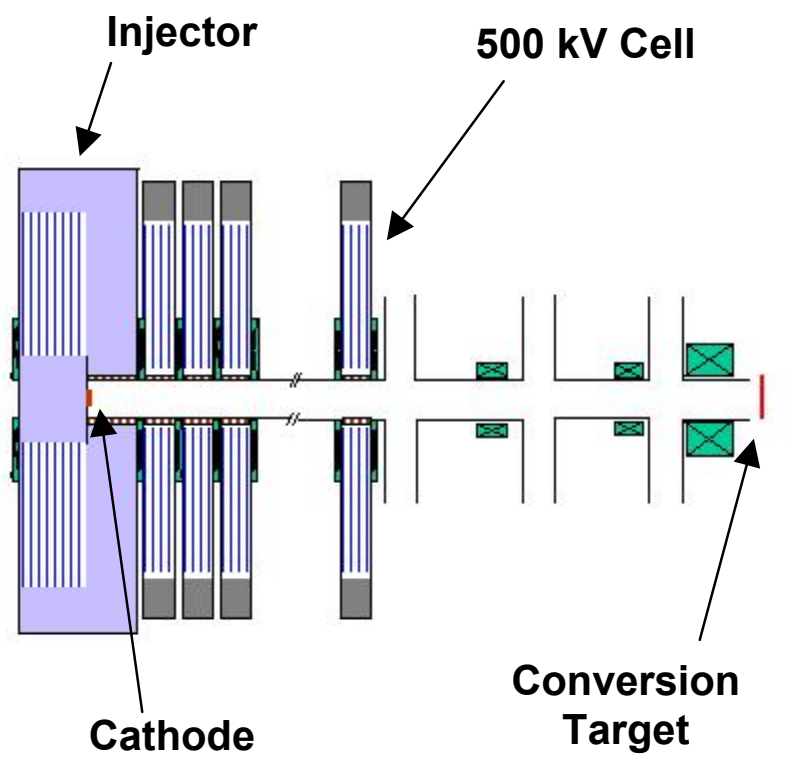

Figure 2. $8 \mathrm{MeV}, 2 \mathrm{kA}$ DWA design.
Although our initial high voltage tests indicate a $(\mathrm{dE} / \mathrm{E})$ of $8-9 \%$, stable transport and minimum corkscrew can be achieved with as much as $(\mathrm{dE} / \mathrm{E}) \sim 14 \%$ because of the high acceleration gradient. Even at this higher limit, the time integrated spot size is $0.6 \mathrm{~mm}$ radius at the final focus magnet. And the on axis dose is $20.5 \mathrm{Rad}$ in air at $1 \mathrm{~m}[4,5]$.

Implementation of the DWA had several technical objectives: achieving the necessary gradients in the vacuum interface and materials, developing low leakage, high specific energy dielectrics, and switching. Because of the topology of the accelerator, the gradients in these components were required to exceed the accelerator gradient; charging was the most stressful condition. These results were achieved and are summarized below.

\section{A. Multi-layer High Gradient Insulators}

A high-gradient insulator (HGI) consists of a series of very thin $(<1 \mathrm{~mm})$ stacked laminations interleaved with conductive planes. This insulator technology was originally conceived and disclosed by Eoin Gray in the early 1980's and resulted from experimental observations that the threshold electric field for surface flashover increases with deceased insulator length. Pulse testing these structures showed a significant improvement in the breakdown electric field compared with conventional insulators (Fig. 3).

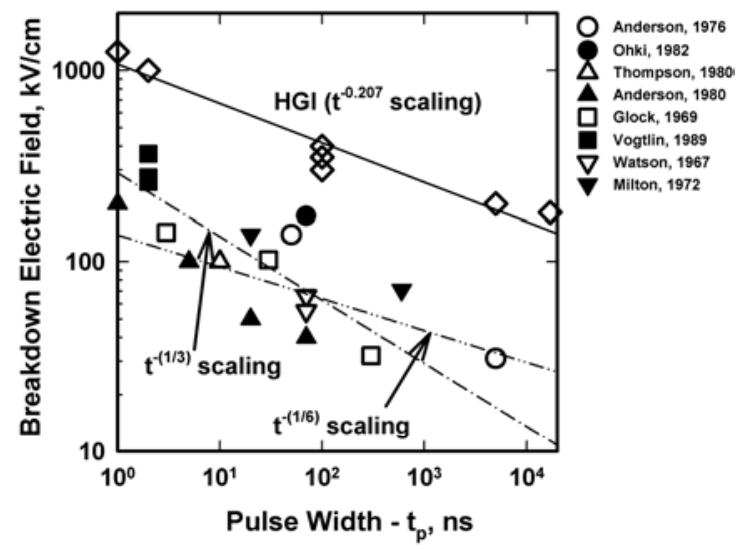

Figure 3. Comparison of multilayer HGI structures with conventional $0^{\circ}$ insulators [6].

Several have postulated that surface flashover results from charge injection into the insulator bulk at an electrode interface and near to the surface. These models predict a $\mathrm{D}^{-0.5}$ scaling, where $\mathrm{D}$ is the overall insulator length. Coincidentally, from our earlier work, we established that these multilayer structures also follow a $\mathrm{d}^{-0.5}$ trend, where $\mathrm{d}$ is the insulator period length. Thus it appears that these high gradient multilayer structures behave as an ensemble of independent conventional 
insulator structures of length $d$. Others have also suggested a resultant modification to the local electrical field that modifies the net trajectory of the electrons, responsible for breakdown, away from the surface.

Regardless of the mechanism, these structure showed robust performance under arced conditions and ranged in surface flashover fields from $>100 \mathrm{MV} / \mathrm{m}$ for $1-3 \mathrm{~ns}$ pulses and $>100 \mathrm{kV} / \mathrm{cm}$ for multi-microsecond pulses to DC. Materials varied from fused silica for UHV applications to plastics treated to reduce the outgassing rates to $2.3 \times 10^{-9}\left(\mathrm{~T}-1 / \mathrm{s}-\mathrm{cm}^{-2}\right)$.

\section{B. Pulse forming Lines and Dielectric Materials}

The pulse forming lines for the DWA are fully integrated into each accelerator cell and result in a very compact system. Work was performed for two geometries: (1) the asymmetric Blumlein and (2) the symmetric Blumlein

The original asymmetric Blumlein, required a large radial extent for a given pulse width and generated a more triangular pulse shape; energy regulation for proper beam transport was difficult to obtain. Further, because of the dissimilar dielectrics, full energy transfer to the load was not possible. The symmetric Blumlein, however, allows full energy transfer. In addition we were able to implement constant impedance pulse forming lines for the generation of a more trapezoidal pulse shape with a flattop. To minimize the radial extent of the lines, a serpentine pattern was used. In this configuration, layerto-layer coupling could occur, but using full 3-d, timedomain electromagnetic field solvers modified specifically for pulsed power applications, allowed us to optimize our designs with a minimum amount of prototyping.

A significant effort focused on the development of engineered dielectrics for the DWA. Initially, pure ceramics were pursued for their high dielectric constant and moderate field gradients. Fabrication was costly and part yield was low. Development of castable composite dielectrics, however, allowed ease of fabrication through a casting process [7]. In addition, tailoring the permittivity to a system specific requirement was possible. Values for the specific energy density as a function of dielectric strength for much of this work are shown in Figure 4. The most notable point, at $250 \mathrm{MV} / \mathrm{m}$ and specific energy density of $>12 \mathrm{~J} / \mathrm{cm}^{3}$, were taken from ten tests conducted with ball electrodes. Enhancement was not taken into account, hence, these values are conservative. Standard deviation of the breakdown electric field was $<0.2$ $\mathrm{MV} / \mathrm{cm}$ or $<1 \%$ of the applied field.

This latter technology is based on a high dielectric constant nano-composite comprised of a polymer resin system and nano-size inorganic particles. The material allows for dielectric moldings with complex shapes or effective encapsulation of electrodes. The polymer/particle slurry does not contain solvents or volatiles and can be net shape formed in 3-dimensional structures.
Component fabrication is achieved by casting the high viscosity slurry and thermally curing the resin system at relatively low temperatures $\left(\sim 100^{\circ} \mathrm{C}\right)$. The physical strength and toughness of the cured dielectric provided a high level of structural integrity. Successful component fabrication is dependent on proper conductor designs and material processing steps including particle dispersion, casting method, thermal and pressure profiles.

The performance of the nano-composite dielectric is achieved through a combination of inorganic powders, dispersants, polymers and cure agents. The polymer system provides the forming characteristics required for encapsulation while the inorganic particles provide an increase in dielectric constant. Proper incorporation of the nano-size particles in the polymer allows for the dielectric constant of the polymer to be increased from approximately 3 to 55 . Further, the voltage stress capability of the polymer is not compromised when using the nano-size powders. The intrinsic dielectric strength of the dielectric has been defined at $250 \mathrm{MV} / \mathrm{m}$.

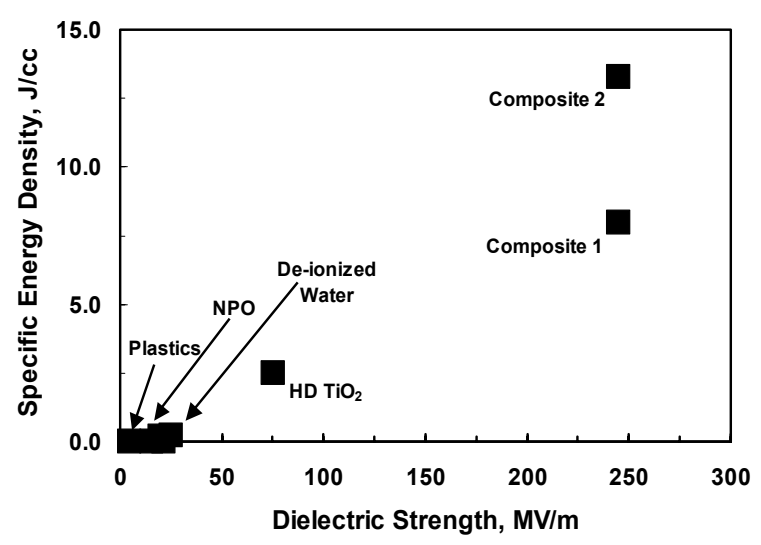

Figure 4. Specific energy density of pulse forming line dielectrics pursued for the DWA

\section{B. Gas Discharge and Photoconductive Switching}

Two switching configurations were tested using RT Duroid as the pulse forming line material. Specific energy density is comparable to most plastics. The first configuration allowed observation of the optical signature of ten laser triggered gas switches integrated into a tenstage Blumlein module. These optical signals were captured on a streak camera and the leading edge was used to measure switching simultaneity (fig. 5). The solid vertical line depicts propagation time for the laser pulse. From these signatures, jitter was determined to be $<1$ ns.

The second configuration used UV-pre-illuminated gas switches. Modifications were made to ensure a low inductance-switching configuration. From this effort we were able to demonstrate a 3-4 ns risetime and $\sim 1$ ns simultaneity on the switch closure time. Output from this 
module was measured to be $90 \mathrm{kV}$ and is usable as an acceleration pulse (fig. 6).

\section{Optical FIDU}

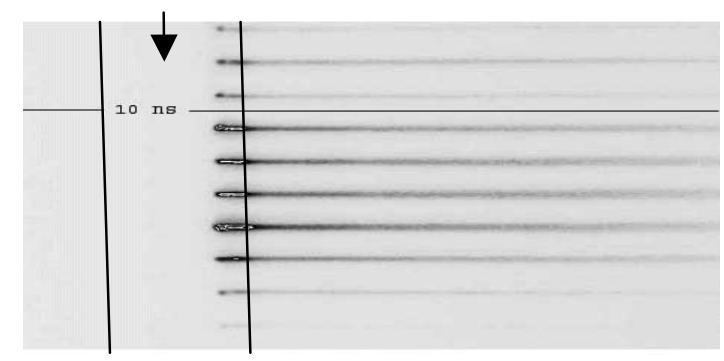

Figure 5. Gas switch triggering simultaneity

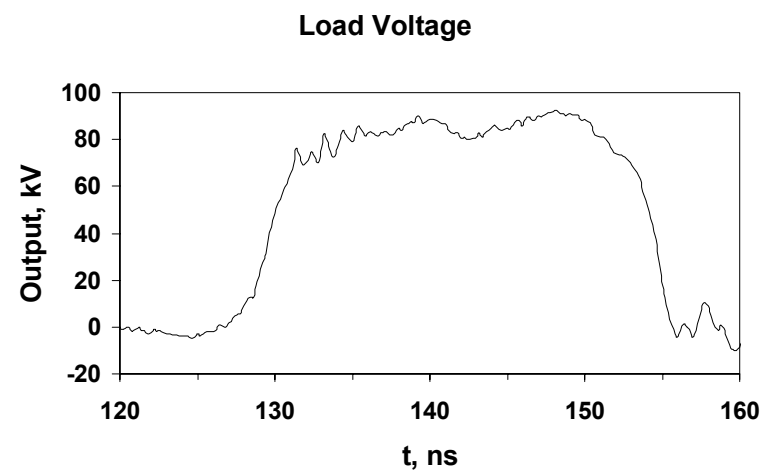

Figure 6. Module output voltage

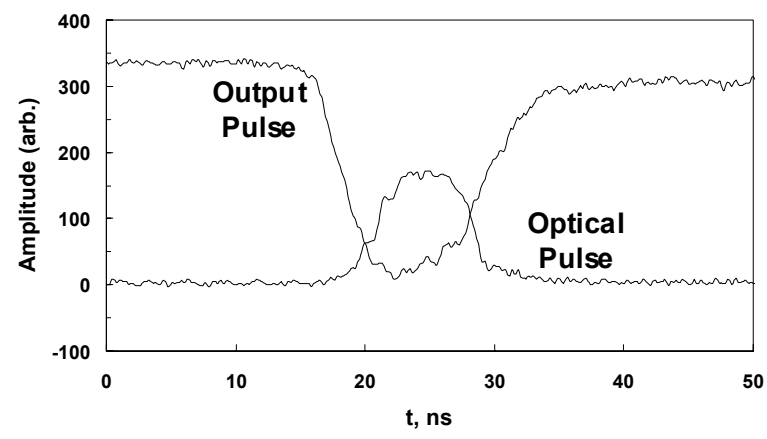

Figure 7. SiC switch. Output (top), optical pulse (bottom)

As part of an ongoing effort for high rep-rate operation, we are pursuing single crystalline $\mathrm{SiC}$ photoconductive switching. In this technology, switching is initiated and maintained with optical energy from a Q-switched $\mathrm{Nd}$ :YAG laser: if operated in a non-avalanche mode, terminating the optical pulse allows recombination of the charge carriers and cessation of conduction. SiC material has multiple advantages over Si and GaAs: a large band gap, high thermal conductivity, and high dielectric strength. The greatest difficulty with this material, however, is the elimination of so-called micro-pipes: small tubular voids that propagate in the direction of crystal growth. These voids lead to breakdown. By taking advantage of alternate cut directions, as in this present data, the material showed excellent dielectric strength and fast recovery (fig. 7).

\section{SUMMARY}

We are developing a compact $8 \mathrm{MeV}, 2 \mathrm{kA}$ accelerator for pulsed radiography. The design will provide a $0.6 \mathrm{~mm}$ radius spot and a dose of 20.5 Rads at $1 \mathrm{~m}$. For this design, we pursued the development of high gradient multilayer insulators (HGI), high specific energy dielectrics for the asymmetric Blumlein pulse forming line, and multi-gap laser triggered low jitter $(<1 \mathrm{~ns})$ gas switches. Testing to date shows insulator surface flashover fields from $>100 \mathrm{MV} / \mathrm{m}$ for $1-3$ ns pulses and $>100 \mathrm{kV} / \mathrm{cm}$ for multi-microsecond pulses to $\mathrm{DC}$, $>10 \mathrm{~J} / \mathrm{cm}^{3}$ specific energy dielectrics, and successful high voltage demonstration of an integrated high voltage module. For burst mode, we successfully demonstrated fast recovery $\mathrm{SiC}$ photoconductive switching.

\section{REFERENCES}

a) University of Missouri, Rolla, MO 65409, USA.

b) On leave from University of Missouri, Columbia, MO 65205, USA.

c) TPL, Inc, 3921 Academy Parkway North, NE, Albuquerque, NM 87109, USA

[1] See for instance http://www.nv.doe.gov/nts/facilities/ ul-a.htm.

[2] S. D. Nelson and B. R. Poole, "Electromagnetic Simulations of Dielectric Wall Accelerator Structures for Electron Beam Acceleration," presented at the 2005 Particle Accelerator Conference, Knoxville, TN (2005).

[3] B. M. Carder, "High-Gradient Compact Linear Accelerator," United States Patent No. 5, 757, 146 (1998). [4] J. McCarrick, G. Caporaso, and Y-J Chen, "Beam Transport in a Compact Dielectric Wall Induction Accelerator for Pulsed Radiography," presented at the 2005 Particle Accelerator Conference, Knoxville, TN (2005).

[5] Y-J. Chen, J. McCarrick, S. D. Nelson, "Transverse Beam Instability in a Compact Dielectric Wall Induction Accelerator," presented at the 2005 Particle Accelerator Conference, Knoxville, TN (2005).

[6] S. E. Sampayan, P. A. Vitello, M. L. Krogh, and J. M. Elizondo, "Multilayer High Gradient Insulator Technology," IEEE Trans. on Diel. And Elect. Insulation, 7(3), 334-339 (2000).

[7] W. Hartman, K. Slenes, and K. Law, "Dielectric Material Including Particulate Filler,' United States patent No. 6,608,760 (2003), other patents pending. 